\title{
¿Es Posible Transformar las Prácticas Evaluativas Mediante el Trabajo Colaborativo? Una Mirada Inclusiva a la Evaluación en Aula
}

\author{
Is it Possible to Transform Assessment Practices Through \\ Collaborative Work? An Inclusive Look at Daily Assessment
}

\author{
Jessica López * \\ Dominique Manghi \\ Pontificia Universidad Católica de Valparaíso, Centro de Investigación para la Educación
} Inclusiva, Chile

\begin{abstract}
El objetivo de este estudio es comprender de qué manera el trabajo colaborativo ha transformado las prácticas evaluativas de dos escuelas públicas de la $\mathrm{V}$ región, hacia el desarrollo de la educación y evaluación inclusiva. La Educación Inclusiva en Chile se abordó inicialmente desde la educación especial, implementando diversas normativas que modificaron las prácticas escolares. El decreto 170/09 regula los Programas de Integración Escolar y señala que profesores de aula y educadores especiales deben enseñar y evaluar juntos el aprendizaje de todos los estudiantes. El diseño metodológico se enmarca en un paradigma epistemológico cualitativohermenéutico. A través de un estudio de casos múltiples, se recoge información mediante análisis documental, entrevistas semiestructuradas y observaciones en aula a dos duplas por escuela. Los principales resultados indican una variedad de prácticas evaluativas realizadas en aula como: adecuación de los instrumentos, prácticas evaluativas y uso de retroalimentación, lo que ha generado condiciones particulares en el trabajo técnico administrativo, prácticas en el aula y la disposición que se han instaurado a medida que los docentes trabajan en conjunto. El trabajo colaborativo como práctica reciente es impulsado por una política que no dialoga con otras menos inclusivas, transformando las prácticas educativas de manera particular en cada caso estudiado.
\end{abstract}

Descriptores: Educación; Evaluación; Inclusión; Retroalimentación, Trabajo en equipo.

The objective of this study is to understand how collaborative work has transformed the evaluative practices of two public schools in the $\mathrm{V}$ region, towards the development of inclusive education and evaluation. Inclusive Education in Chile was initially approached from special education, implementing various regulations that modified school practices. Decree 170/09 regulates the School Integration Programs and indicates that classroom teachers and special educators must teach and evaluate the learning of all students together. The methodological design is framed in a qualitative-hermeneutical epistemological paradigm. Through a multiple case study, information is collected through documentary analysis, semi-structured interviews and classroom observations of two co-teachers per school. The main results indicate a variety of evaluative practices carried out in the classroom such as: adequacy of the instruments, evaluative practices and use of feedback, which has generated particular conditions in technical administrative work, classroom practice and the provision that have been established as teachers work together. Collaborative work is a recent practice prompt by a public policy in relation with others that are les inclusive, transforming educational practices in each case studied.

Keywords: Education; Assessment; Inclusion; Feedback; Team work.

*Contacto: jessica.lopez.g.18@gmail.com

ISSN: 0718-7378

www.rinace.net/rlei/
Recibido: $\quad 15 / 06 / 2020$

$1^{a}$ Evaluación: 19/08/2020

Aceptado: $\quad$ 29/04/2021 


\section{Introducción ${ }^{1}$}

Desde la investigación, la inclusión educativa corresponde al proceso de mejora sistemático que realizan las instituciones educativas, para tratar de eliminar las barreras que limitan el aprendizaje y la participación de los estudiantes en ellas. Esto debe tener particular atención a aquellos más vulnerables; potenciando en las escuelas aquellos procesos que llevan a incrementar la participación y a reducir su exclusión del currículum común, y la comunidad (Booth y Ainscow, 2000).

Las políticas públicas internacionales a las que Chile ha adscrito han invisibilizado las condiciones sociales y económicas de la educación pública de manera situada (Dávila y Naya, 2011), buscando que los sistemas educativos logren la participación de todos sus estudiantes y que a su vez conciban la evaluación respetando las diferentes formas y estilos de aprendizaje del estudiantado, sin considerar la segregación y desigualdad de base. Así y dado el contex to de la educación municipalizada en Chile y de este estudio, se suma un factor muy relevante a la hora de evaluar, Martínez (2009) expone que hay poca conciencia de las dificultades que implica obtener buenos resultados educativos con grupos de alumnos que provienen de un medio social desfavorable.

En Chile, con la incorporación de los Programas de Integración Escolar (PIE), las escuelas de dependencia municipal han enfrentado un gran desafío, el de enriquecer sus prácticas educativas para atender a la diversidad de sus aulas, más aún considerando que en la ley se declara a la Educación Inclusiva como el marco principal desde el cual adscribir a estas normativas (Basualto et al., 2017). La política nacional plantea los PIE como estrategias inclusivas, que implementarán el trabajo colaborativo y, más específicamente, la codocencia entre educadores regulares, educadores diferenciales y otros asistentes de la educación (Ministerio de Educación, 2012). En este escenario, la inclusión se complejiza, sobre todo considerando que muchos directivos y docentes de educación regular creen que la atención a la diversidad es responsabilidad exclusiva de los profesionales ligados a la Educación Diferencial (Bizama et al., 2020; Ossa y Rodríguez, 2014; Tenorio, 2011).

Por una parte, Muñoz y otros (2015) indican que las políticas de inclusión en Chile no han estado acompañadas de un cambio en las prácticas docentes. Por otra, los profesores valoran en gran medida el trabajo colaborativo como el vehículo para el logro de la inclusión en el contexto educacional chileno y el desarrollo del PIE (Bizama et al., 2020). El trabajo colaborativo sería así, una propuesta que desde la normativa, demanda al profesorado a reorganizar y compartir variados ámbitos de su práctica. Hay evidencia sobre los beneficios del trabajo colaborativo para el desarrollo de la educación inclusiva y para mejorar la calidad de los aprendizajes y la evaluación (Cramer y Nevin 2006, citados en Cramer et al., 2010; Villa et al., 2008, entre otros). Así, mientras se mantienen las estructuras segregadoras del sistema educativo chileno (López et al., 2018), hay algunas políticas educativas que buscan garantizar que la evaluación sea accesible para todos los estudiantes, que los procedimientos estén ligados al currículo y promuevan el aprendizaje (Martínez y García, 2017).

De esta manera el trabajo colaborativo podría surgir como una propuesta y estrategia de trabajo en aula que, si bien nace de las normativas ministeriales, se traduce en compartir

\footnotetext{
${ }^{1}$ Este estudio se enmarca en el proyecto SCIA-ANID CIE160009, Centro de Investigación para la Educación Inclusiva
} 
variados ámbitos de la práctica pedagógica, en los cuales se incluyen procesos de evaluación. Es así como, la presente investigación pretende comprender si es posible la transformación de las prácticas evaluativas mediante el trabajo colaborativo incorporado en las escuelas públicas con PIE y como a través del trabajo de co-docencia en duplas, dichas prácticas han interpretado una mirada inclusiva.

\section{Marco conceptual}

\subsection{Educación inclusiva}

A partir de la Conferencia de Salamanca, hace más de 25 años atrás, los diferentes países formularon políticas y normativas de integración escolar, en ellas se asignó un rol importante a la educación especial para el apoyo a los procesos en los que se abrían las puertas de las escuelas a todos los niños y niñas (Blanco y Duk, 2019). Como ya es sabido, en un contexto nacional como internacional existe un acuerdo en torno a que la inclusión educativa es un derecho de todos los alumnos y alumnas, pero también hay consenso respecto a que uno de los principales retos a los que se enfrentan todos los sistemas y comunidades educativas es el de hacer efectivo ese derecho. Es decir, construir sistemas educativos que garanticen la calidad con equidad, lo que equivale a decir que la primera llegue a todos los niños, jóvenes y adultos a lo largo de todas las oportunidades educativas de su vida, sin estar condicionada por razones de salud, de procedencia, género, capacidad económica, etnia, lugar de residencia, orientación afectivo sexual o cualquier otra (Echeita, 2013).

En el caso chileno, la movilidad social solo se ve posible a través de dos estrategias: poseer redes y contactos o credenciales escolares (Araujo y Martucelli, 2015), y la forma de acreditar la calidad de la educación tiene que ver con la capacidad de pago de las familias (Donoso, 2013). La desigualdad está instalada en la sociedad como un valor positivo, ya que se debe competir e intentar con esfuerzo alcanzar los mundos que nos muestra la publicidad, los medios y las redes sociales. Mundos que no serían derecho de todos, sino privilegio de algunos (Cornejo y Reyes, 2008). Estos valores que actualmente han impactado a las escuelas chilenas se consideran opuestos a aquellos valores inclusivos definidos por Booth y Ainscow (2015).

En este marco, la educación inclusiva, más que un tema marginal que trata sobre cómo integrar a ciertos estudiantes a la enseñanza convencional, representa una perspectiva que debe servir para analizar cómo transformar los sistemas educativos y otros entornos de aprendizaje, con el fin de responder a la diversidad de los estudiantes (Leiva, 2013).

Uno de los valores para una Educación Inclusiva es la colaboración en vez de la competencia, en este sentido se considera fundamental el trabajo colaborativo entre los distintos miembros de una comunidad (Booth y Ainscow, 2015; Echeita et al., 2013). Este sería parte del desarrollo de modelos educativos equitativos que afronten con justicia los desequilibrios existentes en la misma, sobre todo en los sistemas de educación pública que en nuestro país atiende a las familias más desfavorecidas.

En el caso de la educación pública chilena de administración municipal, esta recibe sin exclusión a todos los estudiantes sin llevar a cabo un proceso de selección, así se da la paradoja que en materias de aprendizaje los estudiantes reciben una educación de baja calidad o poco pertinente a sus necesidades, intereses y situaciones de vida (Blanco, 2010). Diversos estudios y reportes comparativos, tanto nacionales como internacionales, ponen 
en evidencia que existe relación entre el contexto social, específicamente el nivel socioeconómico, y el logro de los aprendizajes escolares (Donoso, 2013).

En este contexto el trabajo colaborativo y la evaluación de los aprendizajes son centrales para respetar y valorar las diferentes formas y estilos de aprendizaje del estudiantado.

\subsection{Trabajo colaborativo}

La noción de colaboración o trabajo colaborativo ha tenido varias décadas de desarrollo y aparece como una forma de enfatizar lo colectivo. Se declina también a través de las preguntas relativas a la "convivencia", el "clima de aula", la "inclusión" y la "educación ciudadana”, contribuyendo en forjar una retórica educativa global que plantea expectativas para un trabajo colectivo en la escuela (Armijo-Cabrera, 2018). Empíricamente, el trabajo colaborativo ha sido materia de estudio tanto a nivel internacional como nacional. El ámbito internacional lleva más ventaja, puesto que el estudio sobre el "co-teaching" (en español la co-docencia), menciona que en el ámbito educativo, las prácticas de co-docencia les permite a los docentes, compartir experiencias y reflexiones en torno a la enseñanza y recibir retroalimentación continua del quehacer profesional (Strogilos y Stefanidis, 2015).

El desarrollo de estrategias de co-docencia requiere tanto de habilidades individuales (comunicación efectiva, capacidad de resolución de conflictos) y formación profesional docente como también de apoyos administrativos y condiciones contextuales (Strogilos y Stefanidis, 2015), que permitan el trabajo colaborativo entregando facilidades para la planificación y la adecuación curricular necesaria. Además el trabajo en dupla de docentes, entre los educadores regulares y educadores diferenciales, se considera una buena estrategia para abordar la compleja tarea de responder a la diversidad del estudiantado (Basualto et al., 2017).

Durante las últimas décadas y con la finalidad de dar respuesta al problema de la inclusión escolar, se han introducido los Programas de Integración Escolar (PIE) en las escuelas regulares. En el año 2003 se da origen a la Ley 20.201, que además de especificar las regulaciones sobre la subvención escolar preferencial propone la elaboración de un reglamento que se materializa en el decreto supremo $\mathrm{N}^{\circ}$ 170/09 del año 2009 (Marfán et al., 2013), que establece los criterios técnicos respecto a la definición de las Necesidades Educativas Especiales (NEE). Junto con esto, el programa refiere el uso de estrategias orientadas a reforzar el sistema escolar a través del trabajo colaborativo y, más específicamente, la co-docencia entre educadores regulares, educadores diferenciales y otros asistentes de la educación (Ministerio de Educación, 2012). Dentro de sus artículos $\mathrm{n}^{\circ} 86$ y 89 contempla la asignación de 3 horas cronológicas para los profesores de educación regular para la planificación, evaluación y seguimiento de este programa, y las orientaciones para educadores diferenciales en lo que respecta al apoyo a los estudiantes en la sala de clases regular; acciones de planificación, evaluación, preparación de materiales educativos y otros, en colaboración con los profesores de la educación regular (Ministerio de Educación, 2012).

De esta manera, las escuelas de dependencia municipal han enfrentado un gran desafío, el de enriquecer sus prácticas educativas para atender a la diversidad de sus aulas, más aun considerando que en la ley se declara a la Educación Inclusiva como el marco principal desde el cual adscribir a estas normativas (Basualto et al., 2017). 
Es así que desde la reglamentación de la co enseñanza del PIE, se pretende que esta sea una estrategia para proporcionar una ayuda integradora de niños y niñas que generalmente son excluidos del sistema escolar por sus bajos resultados (Figueroa, Sepúlveda, Soto y Yáñez, 2020). Sin embargo, esta práctica inicialmente es coincidente con lo que destaca la teoría, la co-docencia se ejerce bajo la modalidad de "uno enseña, otro circula y atiende a las necesidades de los estudiantes" (Marfán et al., 2013) donde el liderazgo de uno impide una relación de interacción e intercambio mutuo de saberes (Marfán et al., 2013; Ossa y Rodríguez 2014; Pratt, 2014; Strogilos y Stefanidis, 2015).

Ossa y Rodríguez (2014), en sus investigaciones, abordan el trabajo colaborativo bajo una lógica de la gestión curricular y la didáctica, pues se desarrolla mediante un proceso de planificación, instrucción y evaluación colaborativa. Esta última corresponde tanto a las acciones de evaluación curricular como de evaluación de las necesidades educativas especiales. Desde una perspectiva pedagógica, se pretende que la evaluación, sea un aporte para el aprendizaje, de todos y cada uno de los estudiantes, desde una mirada que no excluya a ninguno y respete sus diferentes formas y estilos para aprender (Blanco, 2006).

\subsection{Evaluación del aprendizaje}

La evaluación educativa se relaciona con que las y los profesores puedan emitir un juicio respecto del aprendizaje alcanzado por los alumnos, adoptando acciones de mejora intencionada por los encargados de la enseñanza (Newton, 2007). La evaluación sería aquel proceso en donde se valora la calidad de las respuestas emitidas por los estudiantes, con fines educativos: mejorar sus habilidades y conocimientos y orientarlo para que el aprendizaje ocurra por la enseñanza y que no surja por ensayo y error (Sadler, 1989). El autor además, destaca dentro de su publicación un elemento clave que es la retroalimentación, que es definida como la información proporcionada en torno a cuán exitoso algo se está realizando.

El interés por la evaluación en el aula con propósitos formativos surge con la conciencia de las limitaciones que poseen las pruebas convencionales para tales fines, tanto por el rechazo de los efectos de las pruebas usadas para rendición de cuentas, como por los profundos cambios en las concepciones del aprendizaje y del manejo adecuado de los contenidos (Shepard, 2006). Desde la mirada del modelo tradicional, la evaluación suele ser sumativa y no formativa. Su propósito se centra en una valoración al final de un proceso sin la intención de mejorar los aspectos evaluados, puesto que el afán está en verificar los aprendizajes y no en retroalimentar para la mejora (Jara y Jara 2018).

Popham (2013) define la evaluación formativa como aquel proceso planificado en el que se evidencia de la situación de aprendizaje del alumno y los profesores usan esta información para ajustar sus procedimientos de enseñanza o bien por los estudiantes para ajustar sus formas de aprender. Por consiguiente, la evaluación formativa permitiría no sólo "contar" los aprendizajes sino que también promoverlos (Prieto y Contreras, 2008).

De acuerdo a Shepard (2006), se proponen tres etapas de la evaluación formativa: los profesores recogen informaciones relativas a los progresos y dificultades de aprendizaje del alumno; ellos interpretan estos datos y diagnostican los factores que causan las dificultades; y finalmente, los docentes adaptan las actividades educativas según este diagnóstico.

Biggs (2005) es categórico al manifestar que es la evaluación, y no el currículo oficial, la que determina el aprendizaje de los estudiantes. Desde esta perspectiva, se espera que los 
docentes comprendan que el papel de la evaluación permite orientar, estimular, proporcionar información y herramientas para que los estudiantes progresen en su aprendizaje. Otro aspecto de la evaluación que es necesario considerar es la señalada por Condemarín y Medina (2000), al advertir que se necesita de múltiples estrategias para evaluar el aprendizaje de los estudiantes en su globalidad y complejidad, otorgándole especial relevancia a las actividades cotidianas y significativas que ocurren dentro del aula.

Cualquier cambio en las prácticas de evaluación requiere que las y los profesores tengan "conocimientos claros que den respuesta a: para qué evaluar, qué evaluar, cuándo evaluar, y principalmente, qué debo hacer con los resultados de esa evaluación” (Vera et al., 2017, p. 362). Por ello, se considera conveniente promover la evaluación como herramienta de indagación, análisis y reflexión que lleve a que los docentes realicen una planificación, y que identifiquen estrategias que favorecen o impiden que los alumnos desarrollen los aprendizajes esperados (Martínez y García, 2017).

En síntesis, cabe preguntarse si las prácticas de enseñanza y, en particular, las evaluativas se han modificado con el trabajo colaborativo implementado en los últimos 10 años en escuelas públicas chilenas.

\section{Método}

El presente estudio se enmarca en una investigación desde un paradigma epistemológico cualitativo-hermenéutico, que investiga las representaciones de los sujetos a través de sus discursos y acciones, que emergen de sus vivencias en relación al trabajo colaborativo, y de qué manera se transforman o no las prácticas de evaluación de manera conjunta (Redón y Ángulo, 2017). El diseño corresponde a un estudio de casos múltiples, que permita indagar la realidad social (Stake, 1999).

\section{Participantes}

Esta investigación es parte de un estudio mayor sobre prácticas de aula y escuela para la inclusión (ANID-PIA CIE 160009), por lo que los casos de estudio fueron seleccionados mediante un muestreo intencionado desde la Dirección de Educación de la comuna, con el fin de obtener variadas visiones de manera de diversificar e enriquecer la información.

Los casos de este estudio corresponden a dos escuelas municipales de la comuna de Viña del Mar, a los cuales aludiremos como Caso 1 y Caso 2. Que comparten criterios de homogeneidad al ser escuelas de educación Básica de dependencia municipal, ubicadas en zonas altas de la comuna y atienden a estudiantes pertenecientes a sectores periféricos. Ambas cuentan con PIE y de acuerdo a los resultados de SIMCE, son categorizadas como insuficientes. Además de este criterio de homogeneidad, se aluden a criterios de accesibilidad y heterogeneidad, en cuanto a la selección de los participantes para asegurar diversidad y riqueza de información realizada en conjunto con los jefes de UTP de cada escuela.

En cada escuela, los participantes corresponden a dos duplas de docentes. Cada dupla está conformada por una profesora general básica y una educadora diferencial o psicopedagoga, quienes se desempeñan en cursos de primer ciclo y segundo ciclo de Educación Básica, respectivamente, tal como se muestra en el Cuadro 1. 


\section{Cuadro 1}

Conformación de las duplas que trabajan colaborativamente en cada caso de estudio

\begin{tabular}{|c|c|}
\hline CASO 1 & CASO 2 \\
\hline $\begin{array}{l}\text { Dupla } 1 \text { de trabajo. } \\
\text { Docente de Educación Básica y psicopedagoga. } \\
\text { Cursos de trabajo } 1^{\circ} \text { y } 4^{\circ} \text { básico. } \\
\text { Trabajo colaborativo } 2 \text { años. }\end{array}$ & $\begin{array}{l}\text { Dupla } 1 \text { de trabajo. } \\
\text { Docente de Educación Básica y docente de } \\
\text { Educación Diferencial. Cursos de trabajo } 2^{\circ} \\
\text { básico. } \\
\text { Trabajo colaborativo } 1 \text { año. }\end{array}$ \\
\hline Dupla 2 de trabajo. & Dupla 2 de trabajo. \\
\hline $\begin{array}{l}\text { Docente de Educación Básica y docente de } \\
\text { Educación Diferencial. Cursos de trabajo } 7^{\circ} \text { y } \\
8^{\circ} \text { básico. } \\
\text { Trabajo colaborativo } 2 \text { años. }\end{array}$ & $\begin{array}{l}\text { Docente de Educación Básica y } \\
\text { psicopedagoga. } \\
\text { Cursos de trabajo } 7^{\circ} \text { básico. } \\
\text { Trabajo colaborativo } 1 \text { año. }\end{array}$ \\
\hline
\end{tabular}

\section{Instrumentos de obtención de información}

Se utilizan técnicas etnográficas a través de entrevistas semiestructuradas, realizada a cada uno de los docentes que conformaban las duplas de trabajo; observación participante en aula en 6 periodos pedagógicos durante 2 semanas, y finalmente un análisis documental de libros de clases, actas de reuniones de las duplas, planificaciones y evaluaciones.

\section{Análisis de datos}

Mediante la triangulación de los datos, se realizó un análisis proveniente de 3 fuentes; entrevistas, notas de campos (observaciones) y documentos. Luego se realizó un análisis de contenido categorial. Basándonos en el modelo por pasos del desarrollo deductivoinductivo del análisis de contenido (Cáceres 2003), que incluye la selección del modelo, pre análisis, definición de unidades de análisis, reglas de análisis, códigos de clasificación, para finalmente desarrollar las categorías.

\section{Resultados}

Los resultados se presentarán por caso, desarrollando dos grandes conceptos. Primero lo que las duplas informan sobre la evaluación que realizan en aula y, segundo, de qué manera llevan a cabo el trabajo colaborativo.

\subsection{Caso 1}

A continuación se presentan las categorías emergentes de los docentes del Caso 1 correspondiente a dos duplas, una de primer ciclo y una de segundo ciclo, de la escuela básica municipal con mayor cantidad de estudiantes de la comuna, en un contexto educativo en el marco del PIE.

La primera categoría tiene relación con la evaluación en aula. Las docentes declaran que desde que trabajan colaborativamente realizaron dos grandes cambios; primero en utilización de instrumentos de evaluación, ya que no solo utilizan los instrumentos de evaluación formales como pruebas, sino otras actividades dentro del aula, que logran evidenciar el aprendizaje de sus estudiantes. Por ello destacan la importancia de que la evaluación en cada instancia sea diversificada, priorizando el uso de estrategias para todos los ritmos de aprendizaje de sus estudiantes. 
Los estudiantes trabajan también con material concreto, trabajan con papel lustre, si van a trabajar con valor posicional, si van a trabajar con figuras geométricas, cuerpos geométricos los forman y ellos también por ejemplo trabajo en la pizarra, práctico que les gusta mucho, o si no también videos educativos. (Docente 2, Comunicación Personal, 2018)

De acuerdo a esta cita, se puede percibir que dentro del aula las diversas actividades son consideradas como prácticas evaluativas y no metodologías de trabajo.

El segundo cambio ser relaciona con la retroalimentación y monitoreo de aprendizajes en aula, de acuerdo a lo observado y lo declarado por los docentes, la evaluación, por una parte, se visualiza durante la retroalimentación oral de manera permanente dentro del aula. Así mismo, los docentes logran monitorear los aprendizajes de sus estudiantes, y esto lo materializan en un cuaderno en donde apuntan de manera semanal actividades o trabajos que realizan los estudiantes. Es así que señalan, que ambas actividades tanto de retroalimentación y monitoreo les permite poder corroborar que todos los estudiantes han aprendido, y así mismo para que ellos puedan tomar decisiones respecto a lo que van aprendiendo sus estudiantes y guiarse de eso, para continuar evaluando.

\begin{abstract}
Sé cuándo ellos están aprendiendo no muchas veces por las notas, porque se puede sacar un 5 y por distracción o por no leer bien, se equivocaron. Pero obviamente yo voy viendo en las clases la participación, cuando salen a la pizarra, cuando me preguntan y me dicen: profesora, sabe que vi en internet tal video, profesora sabe que me llama la atención de lo que lei de los incas o de los mayas, pero me doy cuenta en el proceso, con el trabajo de ellos diariamente. (Docente 1, Comunicación Personal, 2018)
\end{abstract}

Las duplas de docentes de manera paulatina han incorporado nuevos elementos evaluativos, diferentes a los que siempre son utilizados, como es la diversificación de instrumentos evaluativos, utilizando una gamma de estrategias con foco en ser una evaluación diferenciada para cada estudiante, con foco en la retroalimentación para verificar los aprendizajes de los estudiantes.

La segunda categoría, se relaciona con el Trabajo colaborativo, este se construye a partir de las modificaciones en el proceso de co enseñanza, luego la planificación y finalmente la evaluación en conjunto.

En el proceso de co enseñanza en el aula, los docentes informan que se han ido paulatinamente modificando los roles que posee cada una de las duplas en el aula. Es decir, comenzó siendo un trabajo de apoyo, en donde el educador de enseñanza general básica guiaba la clase y el educador diferencial y/o psicopedagoga apoyaba a los estudiantes de individual, esto se modificó y ahora ambos docentes realizan la clase en conjunto.

Por otra parte, las reuniones de trabajo colaborativo, han sido de gran utilidad para que los docentes puedan planificar y evaluar juntos. Respecto a las reuniones, ellos declaran que las decisiones se toman en conjunto:

Las decisiones las tomamos en conjunto. Ayer estuvimos de pre kínder a cuarto básico, viendo lo mismo: las unidades y lo que se va a trabajar, estamos empapándonos de eso, todo depende de la asignatura, del contenido, etc., si se toma oral o distinto. Con X hacíamos trabajo colaborativo, porque conversábamos sobre cómo evaluar y qué hacer, pero no dan las horas, porque son ocho en aula, generalmente, son cuatro en lenguaje y cuatro en matemáticas. Son muy poquitas horas. (Docente 2, Comunicación Personal, 2018)

Esto se ve reflejado en la evaluación que realizan en aula, ya que han innovado en el uso de instrumentos evaluativos utilizando trabajos en clases, disertaciones, actividades al aire libre, durante el trabajo en aula. La clave que permite modificar estas prácticas tiene que 
ver con el constante monitoreo que se realizan a los estudiantes, la toma de decisiones que toman en conjunto los docentes mediante el trabajo colaborativo, para poder modificar y mejorar sus prácticas evaluativas.

\subsection{Caso 2}

A continuación, se presentan las categorías emergentes de los docentes del Caso 2 correspondiente a dos duplas, una de primer ciclo y una de segundo ciclo, de la escuela básica municipal que cuenta con una matrícula baja, lo que lo hace tener cursos fusionados, es decir dos cursos en unos que no alcanzan los 5 a 10 estudiantes, lo cual cambia la dinámica de trabajo de los docentes respecto al trabajo curricular. Los factores contextuales que dificultan el trabajo en aula, son baja asistencia y atrasos en clases por parte de los estudiantes, todo en un contexto educativo en el marco del PIE.

Respecto a la primera categoría de evaluación en aula, las duplas de docente reportaron tres elementos centrales; la evaluación es situada es decir, considera el contexto del estudiante, segundo se utiliza como práctica habitual el juego y, finalmente, la calificación a través de una nota.

La forma de evaluar de las duplas del caso 2 se encuentra en estrecha relación con la realidad social de la educación municipal. En el marco del concepto de evaluación situada que poseen los docentes, su definición se genera a partir del contexto social común de los estudiantes.

El tema de evaluar hay que fijarse bien en el tipo de niños que tenemos y sus estilos de aprendizaje, aparte el grado de vulnerabilidad que tienen también, de dónde vienen, fijarse bien en eso y tratar de adaptar bien el contenido a ellos para que puedan aprender. (Docente 1, Comunicación personal, 2019)

El juego es otro de los procesos evaluativos, a través del cual recogen información dentro del aula para conocer el progreso curricular y el logro de objetivos clase a clases.

lo ideal es brindarle el contexto del juego para poder realmente hacer una evaluación más formativa, estamos en esta actividad, le propuse hacer esto y lo logró, pero a través de una observación más minuciosa, más allá de algo tan pauteado. (Docente 2, Comunicación personal, 2019)

Las duplas han acordado usar la calificación para otorgar la posibilidad a los estudiantes de acumular notas de trabajos realizados en clases. Esto es posible evidenciarlo en las prácticas de aula, en donde prima la utilización de instrumentos evaluativos con foco en la calificación; a través de los trabajos en clase con nota acumulativa, en el canje de una nota por otra, en las actividades en aula con puntajes acumulativos, entre otras. Respecto a la evaluación calificada, es decir, con nota las duplas mencionan

\footnotetext{
Si yo veo un rojo conversamos qué podemos hacer, acá se niega mucho la realidad. Tengo que aplicar un poco la sensibilización, explicar "mire este niño tiene 17 años va en séptimo... por algo es”, entonces hay que hacer algo para que pueda mejorar su nota. (Docente 3, Comunicación Personal, 2019)
}

De esta manera la nota cobra suma importancia, y emerge como una manera de motivar, e implicar al estudiantado en el aprendizaje. Bajo esta modalidad, es que se otorga la posibilidad de realizar diversos trabajos dentro del aula, que serán con nota acumulativa con el fin de mejorar el promedio final.

En síntesis, adaptar y modificar la práctica evaluativa para considerar la diversidad de estudiantes, incluye en el caso 2 un conjunto de factores a través del trabajo colaborativo. 
Estos logros asumen una diversificación de prácticas evaluativas en aula, con foco en atender a todos los estudiantes de acuerdo a sus particularidades y ritmos de aprendizaje.

Respecto a la segunda categoría Trabajo colaborativo, las duplas de docente reportaron, primero que el trabajo colaborativo está enfocado en calificar o cumplir con lo propuesto por el plan curricular. Desde el ámbito del aprendizaje, su uso posee relación con ver el progreso de los estudiantes, en función de si han mejorado o no las notas, esto indicaría si ha aprendido más y ha avanzado en relación a cada actividad que realizan en el aula.

En relación a las modificaciones que ha tenido el trabajo colaborativo a lo largo del tiempo. En primero lugar, respecto al rol de la educadora diferencial dentro del aula, ha pasado de ser un trabajo de apoyo a un trabajo de co-docencia, hacia uno en que juntos trabajan en las diversas actividades que fueron planificadas, realizando la clase en conjunto. En segundo lugar, se evidencian mejoras en lo que respecta a innovar en la elaboración de instrumentos evaluativos. Este trabajo se ha gestado con el tiempo y mayoritariamente se ha trabajado de manera reflexiva en momentos de coordinación, que desde lo técnico administrativo se asume como una práctica consolidada de reuniones para poder planificar las actividades en aula. Esta cooperación entre docentes deja en manifiesto a través del relato de ellos mismo, y a través de las actas de reunión, de un proceso que en un comienzo señalan fue difícil de afianzar, pero en el tiempo han visualizado ellos mismos los beneficios de trabajar colaborativamente.

\section{Discusión y conclusiones}

Lo que se describe a continuación, son las modificaciones que han generado las duplas de trabajo en sus prácticas evaluativas, que desde que trabajan en conjunto declaran comprender la evaluación de una manera diferente.

La evaluación es entendida por las duplas como un proceso, que posee características particulares en cada estudio de caso. Si bien en el caso 1, la evaluación es percibida de una manera más flexible, adaptable y que se mejora constantemente, con foco en los estudiantes y con rasgos de evaluación formativa; en el caso 2 además, es un proceso situado en el contexto del estudiante, es calificado, y se enfoca en el progreso del alumno, por lo tanto, se aproxima más al enfoque de la evaluación de carácter sumativo. La teoría nos informa de un elemento central de la práctica docente, que es la evaluación que el profesor hace del progreso de sus alumnos para alcanzar buenos niveles de aprendizaje. Según el uso que se haga de los resultados, puede tener el enfoque denominado formativo si el resultado se utiliza para orientar al alumno para que su aprendizaje mejore y para que el profesor tome decisiones sobre su enseñanza; o un enfoque sumativo si se limita a llegar a una conclusión sobre el estudiante, como la de promoverlo al grado superior o reprobarlo, asignarle una calificación u otra (Martínez, 2012).

Continuando con esta idea respecto a los tipos de evaluación, el caso 1 ha adaptado sus prácticas con el fin de otorgar más oportunidades de aprendizaje a sus estudiantes, que responden en alguna medida a lograr que todos puedan aprender a su ritmo. En aula este proceso evaluativo es motivante y además el docente genera una retroalimentación constante durante cada clase, que es para todos los estudiantes. Se crean instancias en donde los alumnos pueden participar y ser retroalimentados de manera oral por el docente. Desde esta perspectiva la evaluación que realiza el caso 1 tiene características formativas ya que nos informa sobre cómo se recupera el valor de la evaluación. Esta es una 
evaluación más centrada en las necesidades de los estudiantes, y supeditada a constantes cambios debido a la toma de decisiones por parte de los docentes, y la constante retroalimentación que se desarrolla en aula.

Las duplas de docentes, pueden evidenciar si sus estudiantes están aprendiendo a través del proceso de retroalimentación que hacen en clases, en donde monitorean las actividades en aula. Así es como recogen información de cómo los estudiantes están aprendiendo, y deciden en función a esta cómo adaptar sus prácticas evaluativas. La información más relevante de este caso 1 es que a lo largo del tiempo han innovado en el uso de instrumentos evaluativos utilizando trabajos en clases, disertaciones, actividades al aire libre, durante el trabajo en aula, con el propósito de recoger información sobre como aprenden los alumnos. La clave que permite modificar estas prácticas tiene que ver con el constante monitoreo que se realizan a los estudiantes, otorgándole un sentido de aprendizaje, más que de calificación, lo que le da un carácter de evaluación formativa.

El caso 2, por otra parte, sus prácticas evaluativas son actividades que deben ser calificadas, mediante esa nota pueden visualizar el progreso de los estudiantes en función de sus aprendizajes. Es además una evaluación de progreso porque siempre se otorga calificaciones sumativas para poder "subir" las notas y, de manera importante, posee un sesgo socio cultural, al entender que las actividades se adecuan por el grado de vulnerabilidad del estudiantado y no por sus capacidades. Este criterio de evaluación le otorga un sentido más social, por una parte, busca motivar al estudiante a través de la nota $y$, por otra, intenta comprometerlo en el trabajo y diversas actividades en aula.

Vale la pena destacar que en ambos casos estudiados las duplas vivencian contradicciones de manera cotidiana. Por una parte, los casos estudiados en cuanto a las prácticas de evaluación se orientan al desarrollo de las potencialidades y habilidades de los estudiantes, incluso a darles seguridad y un sentido de pertenencia a los estudiantes. El trabajo colaborativo inmerso en un contexto de valores inclusivos y excluyentes contradictorios, genera prácticas evaluativas muy particulares siendo algo beneficioso no solo para los estudiantes, sino también para el desarrollo de potencialidades y habilidades de los propios profesores y profesionales de las duplas que realizan co-docencia (Martínez y García, 2017).

Por otra parte, recordando que ambas escuelas se encuentran calificadas como insuficientes y con la amenaza constante de cierre del establecimiento, los docentes se encuentran en posiciones complejas, en las cuales se enfrenta cotidianamente a decidir si educan o enseñar para los exámenes estandarizados (Darling-Hammond, 2004). Entonces junto con sus transformaciones en las prácticas evaluativas conviven con el uso de instrumentos evaluativos de carácter cuantitativo, a lo que se suma la predominancia de aquellos ministeriales como la evaluación progresiva de lenguaje y matemáticas en $2^{\circ} \mathrm{y}$ $7^{\circ}$ básico respectivamente, en donde de manera psicométrica se mide el aprendizaje de los estudiantes, así como también el SIMCE, evaluación institucional y obligatoria.

El trabajo colaborativo se plantea como una de las principales estrategias organizacionales y curriculares utilizadas desde un enfoque inclusivo para el aprendizaje de todos los estudiantes (Rodríguez, 2012). Es importante destacar que a 10 años de la implementación de las normativa se evidencian avances en relación al trabajo en aula que realizan las duplas mediante el trabajo colaborativo. A su vez, destacamos las transformaciones que lograron generar las duplas de docentes en dos aspectos: primero en relación al trabajo técnico administrativo relacionado a la implementación del decreto, esto consiste; en una 
práctica de trabajo colaborativo mediante reuniones periódicas, que tienen las duplas de docentes. Desde la Ley, ambas escuelas han cumplido el aspecto técnico solicitado por la normativa, han otorgado horas para esta función para ambos miembros de la dupla: en donde los mismos docentes generan horarios de reuniones de manera semanal, o mensual, de acuerdo a su disponibilidad horaria. En este contexto las duplas de docentes se enfrentan al factor tiempo como un obstáculo para ejercer dicha práctica, esto se traduce en dos aspectos. Concretamente los docentes mencionan que es difícil hacer coincidir los horarios de ambos para poder realizar reuniones periódicas, o juntarse a realizar planificaciones; una vez que logran coordinar un horario en común, cronológicamente son alrededor de 45 minutos que otorgan a estas reuniones, lo cual es insuficiente para poder planificar y realizar evaluaciones en conjunto. Más allá de solo cumplir con la normativa respecto a las reuniones semanales, los docentes efectivamente trabajan colaborativamente en el escaso tiempo que pueden reunirse.

Como segunda transformación se mencionan las prácticas evaluativas, que reconocen el contexto vulnerable de las escuelas municipales de Chile y sus estudiantes, y logran adaptar y modificar sus evaluaciones en función de las necesidades específicas de sus estudiantes. En contraste con los mecanismos de evaluación establecidos en Chile los cuales tienden a orientarse casi exclusivamente a los resultados de aprendizaje, utilizando el SIMCE, como su principal indicador (Torche et al., 2015). Este tipo de evaluación estandarizada no considera factores personales o ritmos de aprendizaje de los estudiantes, ni el contexto situado en el que se da el aprendizaje, en este caso, entornos de desigualdad y extrema pobreza.

Cuando las evaluaciones son iguales para todos, no son flexibles y tampoco consideran el trabajo en aula ni las prácticas diarias de evaluación, se produce un dilema, ya que las políticas educativas de inclusión y la implementación del trabajo colaborativo en los PIE a nivel nacional, han otorgado lineamientos para la diversificación de procedimientos evaluativos, a través del decreto 83/2015 sobre adecuación curricular y diversificación de la enseñanza. Sin embargo hay una clara tendencia emanada desde el Ministerio de Educación a contabilizar los aprendizajes a través del SIMCE, y a su vez catalogar a las escuelas en función de ello.

Estos resultados afectan directamente a nuestros dos casos de estudio. Por una parte, invisibilizan las prácticas de evaluación en aula que realizan los docentes de manera diaria, con foco en atender a la diversidad de estudiantes que se encuentran en situación de riesgo y vulnerabilidad. Por otra parte, las categorizaciones de las escuelas en función de sus resultados en estas mediciones siguen la lógica de la sanción, funcionando como una amenaza permanente que puede incluso llegar al cierre de la escuela. Ambos efectos son contradictorios a los valores inclusivos (Booth y Ainscow, 2015) que busca la política pública. Los esfuerzos por potenciar el aprendizaje y la participación de todos y cada uno de los estudiantes deben acompañarse de un sistema de evaluación y políticas educativas que respeten $\mathrm{y}$ valoren las diferencias individuales para evitar profundizar las desigualdades (Murillo y Duk, 2012). 


\section{Agradecimientos}

Este estudio se desarrolló previo a la pandemia en el marco del proyecto SCIA-ANID CIE160009, Centro de Investigación para la Educación Inclusiva, Línea 4: Prácticas de Aula y Escuela para la Inclusión.

Se agradece a las comunidades educativas Escuela Santa María de Agua Santa y Colegio Miraflores, de la comuna de Viña del Mar que nos abrieron las puertas para investigar.

En memoria de Alexandra, profesora participante en este estudio.

\section{Referencias}

Araujo, K. y Martuccelli. D. (2015) La escuela y la cuestión del mérito: Reflexiones desde la experiencia chilena. Educação e Pesquisa, 41, 1503-1518.

Armijo Cabrera, M. (2018). La colaboración en Educación, trayectorias de un concepto. Siglo XIX-XXI.

Basualto, P. Durán, C. Miranda, P. y Urbina, C. (2017). Prácticas de co-docencia: El caso de una dupla en el marco del programa de integración escolar en Chile. Revista Estudios Pedagógicos, $2,355-374$.

Biggs, J. (2005). Calidad del aprendizaje universitario. Narcea.

Bizama, M. Careaga, M. y Sagredo, E. (2020) Análisis crítico acerca de políticas públicas relacionadas con inclusión e integración escolar en Chile. Revista Espacios, 41(9), 6-15.

Booth, T. y Ainscow, M. (2000). Index for inclusion. Developing leaning and participation in schools. CSIE.

Booth, T. y Ainscow, M. (2015). Guía para la educación inclusiva. Desarrollando el aprendizaje y la participación ciudadana en los centros escolares. OEI-FUHEM.

Blanco, R. (2006). La equidad y la inclusión social: Uno de los desafíos de la educación y la escuela. REICE. Revista Iberoamericana sobre Calidad, Eficacia y Cambio en Educación, 4(3), 1-15.

Blanco, R y Duk, C (2019). El legado de la conferencia de salamanca en el pensamiento, políticas y prácticas de la educación inclusiva. Revista Latinoamericana de Educación Inclusiva, 13(2) 2543. http://doi.org/10.4067/SO718-73782019000200025.

Blanco, R. (2010). Monográfico: El derecho de todos a una educación de calidad. Revista Latinoamericana de Educación Inclusiva, 4(2), 25-153.

Cáceres, P. (2003). Análisis cualitativo de contenido: Una alternativa metodológica alcanzable. Revista Psicoperspectivas, 2(1), 53-82.

Condemarín, M. y Medina, A. (2000). Evaluación auténtica de los aprendizajes. Un medio para mejorar las competencias en lenguaje y comunicación. Andrés Bello.

Cramer, E., Liston, A., Nervin, A. y Thousand, J. (2010). Co-teaching in urban secondary school. Districts to meet the needs of all teachers and learners: Implications for teacher education reform. International Journal of Whole Schooling, 6(2),59-76.

Dávila, P. y Naya, L. (2011). La defensa de los derechos de la infancia en América Latina desde la perspectiva legal una visión educativa. Revista Educación XXI, 14(1), 201-221.

Darling-Hammond, L. (2004). Standards, Accountability, and School Reform. Teachers College Record, 106(6), 1047-1085. 
Donoso, S. (2013). Desplazamiento cotidiano de estudiantes entre comunas de Chile: Evidencia y recomendaciones de política para la nueva institucionalidad de la Educación Pública. Revista EURE, 39(116), 39-73.

Echeita, G. (2013). Inclusión y exclusión educativa. De nuevo 'voz y quebranto’. REICE. Revista Iberoamericana sobre Calidad, Eficacia y Cambio en Educación, 6(2), 9-18.

Figueroa, I. Sepúlveda, G. Soto, J. y Yáñez, C (2020). Coenseñanza entre docentes de educación general básica y educadoras diferenciales: Incidentes críticos de la práctica colaborativa en proyectos de integración educativa. Revista de Investigación Educacional Latinoamericana, $57(1), 1-15$

Jara, M. Jara, P. (2018). Concepciones y prácticas evaluativas declaradas por los docentes en respuesta a las necesidades educativas especiales de carácter permanente. Revista Latinoamericana de Educación Inclusiva, 12(2), 59-77. https://doi.org/10.4067/S0718-73782018000200005.

Leiva, J. (2013). De la integración a la inclusión: Evolución y cambio en la mentalidad del alumnado universitario de educación especial en un contexto universitario. Actualidades Investigativas en Educación, 13(3), 1-27.

López, V., González, P., Manghi, D., Ascorra, P., Oyanedel, J. C., Redón, S., ... y Salgado, M. (2018). Políticas de inclusión educativa en Chile: Tres nudos críticos. Archivos Analíticos de Políticas Educativas, 26(157), art. 6. http://doi.org/10.14507/epaa.26.3088

Marfán, J., Castillo, P., González, R. y Ferreira, I. (2013). Análisis de la complementación de los programas de integración escolar (PIE) en establecimientos que han incorporado estudiantes con necesidades educativas especiales transitorias (NEET). Ministerio de Educación.

Martínez, F. (2009). Evaluación formativa en aula y evaluación a gran escala. Hacia un sistema más equilibrado. Revista REDIE, 11(2), 11-21.

Martínez, F. (2012). La evaluación formativa del aprendizaje en el aula en la bibliografía en inglés y francés. Revista Mexicana de Investigación Educativa, 17(54), 849-875.

Martínez, C y García, I. (2017). Análisis de las prácticas evaluativas inclusivas de tres docentes de educación preescolar en México. Revista Latinoamericana de Educación Inclusiva, 11 (1), 225243.

Ministerio de Educación. (2005). Política nacional de educación especial: Nuestro compromiso con la Diversidad. MINEDUC.

Ministerio de Educación. (2012). Orientaciones técnicas para programas de integración escolar. MINEDUC.

Muñoz, M. López-Cruz, M. y Assaél, J. (2015). Concepciones docentes para responder a la diversidad: ¿Barreras o recursos para la inclusión educativa? Revista Psicoperspectivas, Individuo y Sociedad, 14(3) 68-79.

Murillo, F. y Duk, C. (2012). Una evaluación inclusiva para una educación inclusiva. Revista Latinoamericana de Educación Inclusiva, 6(1), 11-13.

Newton, P. (2007) Clarifying the purposes of educational assessment. Assessment in Education. Principles, Policy y Practice, 14(2), 149-170.

Ossa, C. y Rodríguez, F (2014). Valoración del trabajo colaborativo entre profesores de escuelas básicas de Tomé, Chile. Estudios Pedagógicos, 4O(2), 303-319.

Popham, W. (2013) Evaluación transformativa: El poder transformador de la evaluación formativa. Narcea. 
Prieto, M. y Contreras, G. (2008). Las concepciones que orientan las practicas evaluativas de los profesores: un problema a develar. Estudios Pedagógicos, 24(2), 69-98.

Redón, S. y Ángulo, J. (2017). El estudio de caso. En Investigación cualitativa en educación. Miño y Dávila.

Rodríguez, F. (2012). La percepción del trabajo colaborativo en la gestión curricular de profesores de educación regular y educación especial en Programas de Integración Escolar de la comuna de Tomé (Trabajo Fin de Máster). Universidad del Bío-Bío.

Sadler, D. (1989). Formative assessment and the design of instructional systems. Instructional Science, 18, 119-144.

Shepard, L. A. (2006). Classroom assessment. En R. L. Brennan (Ed.), Educational Measurement, 4, 623-646.

Stake, R. (1999). Investigación con estudio de casos. Morata.

Strogilos, V. y Stefanidis, A. (2015). Contextual antecedents of co-teaching efficacy: Their influence on students with disabilities' learning progress, social participation and behaviour improvement. Teaching and Teacher Education, 47, 218-229.

https://doi.org/10.1016/j.tate.2015.01.008

Tenorio, S. (2011). Formación inicial docente y necesidades educativas especiales. Estudios Pedagógicos, 24, 249-265.

Torche, P., Martínez, J., Madrid, J. y Araya, J. (2015). ¿Qué es la educación de calidad para directores y docentes? Revista Calidad en la Educación, 43, 103-135.

Vera, A., Poblete, S. y Díaz, C. (2017). Percepción de los docentes chilenos sobre sus perspectivas, habilidades y prácticas evaluativas en el aula. Estudios Pedagógicos, 43(3), 361-372.

Villa, R., Thousand, J. y Nevin, A. (2008). A guide to co-teaching. Practical tips for facilitating student learning. Corwin Press.

\section{Breve CV de las autoras}

\section{Jessica López}

Profesora de Educación Diferencial y Magister en Educación mención Evaluación Educativa (PUCV). Profesora en Programa de Integración Escolar en establecimiento dependiente de la corporación Municipal de Valparaíso. ORCID: https:/ / orcid.org/ooooo001-6928-9795. Email: jessica.lopez.g.18@gmail.com

\section{Dominique Manghi}

Doctora en Lingüística (PUCV) y Licenciada en Educación (UMCE), es profesora adjunta de la Escuela de Pedagogía de la Pontificia Universidad Católica de Valparaíso. Investigadora principal del Centro de Investigación para la Educación Inclusiva. Sus líneas de investigación son el análisis multimodal del discurso, lingüística y semiótica educacional, alfabetización semiótica, prácticas educativas y educación inclusiva. ORCID: https://orcid.org/0000-0002-0278-9899. Email: dominique.manghi@pucv.cl 